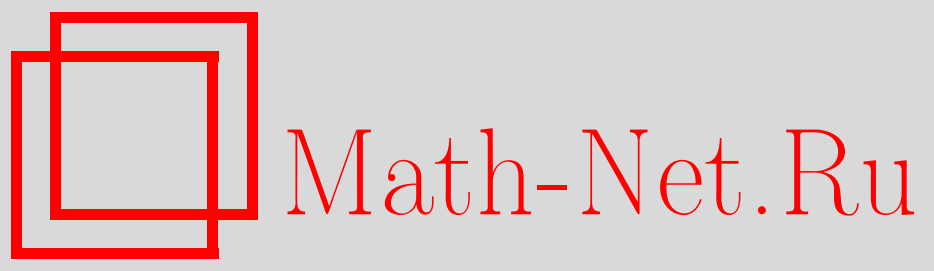

С. А. Гриценко, Д. Б. Демидов, О нулях линейных комбинаций специального вида функций, связанных с $L$ функциями Гекке мнимых квадратичных полей, лежащих на коротких промежутках, Совр. пробл. матем., 2013, выпуск 17, 164-178

DOI: https://doi.org/10.4213/spm49

Использование Общероссийского математического портала Math-Net.Ru подразумевает, что вы прочитали и согласны с пользовательским соглашением http: //www . mathnet.ru/rus/agreement

Параметры загрузки:

IP : 54.92 .164 .108

26 апреля 2023 г., $17: 16: 47$

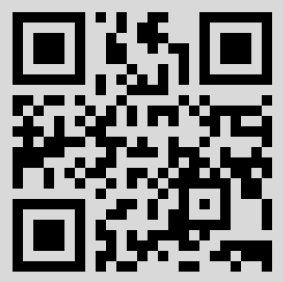




\title{
О нулях линейных комбинаций специального вида функций, связанных с $L$-функциями Гекке мнимых квадратичных полей, лежащих на коротких промежутках
}

\author{
С. А. Гриценко, Б. Д. Демидов \\ Белгородский государственный университет
}

Посвящается светлой памяти Анатолия Алексеевича Карацубы

\section{1. Введение}

Пусть $N_{0}(T)$ - число нулей $\zeta(1 / 2+i t)$ на промежутке $(0, T]$.

В 1921 г. Харди и Литтлвуд [1] доказали, что

$$
N_{0}(T) \geqslant c_{1} T, \quad c_{1}>0-\text { абсолютная постоянная. }
$$

В 1942 г. Сельберг [2] получил правильную по порядку оценку $N_{0}(T)$ :

$$
N_{0}(T) \geqslant c_{2} T \log T, \quad c_{2}>0-\text { абсолютная постоянная. }
$$

Для арифметических рядов Дирихле, удовлетворяющих функциональному уравнению риманова типа, но не имеющих эйлерова произведения, правильные по порядку нижние оценки для числа их нулей на отрезках критической прямой $\operatorname{Re} s=1 / 2$ пока не получены.

Первым, кто показал, что на критической прямой лежит аномально много нулей арифметического ряда Дирихле без эйлерова произведения, был Воронин, который в 1980 г. доказал [3], что

$$
N_{0}(T, f)>c_{3} T \exp \left(\frac{1}{20} \sqrt{\log \log \log \log T}\right),
$$

где $N_{0}(T, f)$ - число нулей $\rho$ функции Дэвенпорта-Хейльбронна $f(s)$ таких, что $\operatorname{Re} \rho=1 / 2$, $0<\operatorname{Im} \rho \leqslant T, c_{3}>0$ - абсолютная постоянная.

В 1989 г. А. А. Карацуба [4] разработал новый метод оценок снизу числа нулей некоторых рядов Дирихле на отрезках критической прямой, с помощью которого доказал следующее неравенство, существенно усилив тем самым результат Воронина (1):

$$
N_{0}(T, f) \geqslant T \sqrt{\log T}(\log T)^{-\varepsilon},
$$

где $\varepsilon$ - сколь угодно малое положительное число, $T>T_{0}(\varepsilon)>1$.

Пусть $Z(t, \chi)=e^{i \theta(t, \chi)} L(1 / 2+i t)$, где функция $\theta(t, \chi)$ подобрана так, что $Z(t, \chi)$ вещественна при вещественных $t$. Тогда $Z(t, \chi)$ представляет собой аналог функции Харди (см., например, [5; гл. 2]).

Пусть

$$
G(t)=a_{1} Z\left(t, \chi_{1}\right)+\cdots+a_{N} Z\left(t, \chi_{N}\right),
$$

где $a_{1}, \ldots, a_{N}-$ произвольные вещественные числа.

Работа выполнена при поддержке ФЦП «Научные и научно-педагогические кадры инновационной России» (госконтракт 14.A18.21.0357). 
В 1991 г. А.А. Карацуба [6] поставил и решил своим методом 1989 г. задачу о нижней оценке числа нулей $G(t)$ на отрезке $(0, T]$.

Эту задачу мы называем задачей $A$. А. Карачубъ.

В 1991 г. Сельберг [7] определил класс $S$ рядов Дирихле и высказал ряд гипотез о свойствах функций из $S$.

В класс $S$ помимо дзета-функции Римана, $L$-функций Дирихле входят и другие арифметические ряды Дирихле, в частности, такие ряды $F(s)$, приближенные функциональные уравнения которых содержат порядка $|\operatorname{Im} s|$ слагаемых. В соответствии с классификацией, предложенной в [8], такие ряды из класса Сельберга имеют степень 2.

В 1996 г. Гриценко рассмотрел вопрос о числе $N_{0}(T, f)$ нулей на отрезке $[0, T]$ функции

$$
f(t)=\sum_{j=1}^{N} a_{j} Z\left(t, F_{j}\right)
$$

где $a_{j}$ - произвольные вещественные числа, а $Z\left(t, F_{j}\right)$ - аналоги функции Харди, соответствующие функциям $F_{j}(s)$ из класса Сельберга степени $2, j=1, \ldots, N$.

В [9] доказано, что при условии справедливости некоторых гипотез, являющихся гипотезами Сельберга и их слегка усиленными вариантами, справедлива оценка

$$
N_{0}(T, f) \gg T \exp \{\sqrt{\log \log \log T}\} .
$$

Определим подкласс класса Сельберга, состоящий из рядов Дирихле степени 2. Пусть $K=\mathbb{Q}(\sqrt{d})$ - мнимое квадратичное поле, $\mathbb{Z}_{K}-$ кольцо целых алгебраических чисел $K$. Следуя терминологии книги [5], будем называть характерами Гекке групповые характеры группы классов идеалов кольца $\mathbb{Z}_{K}$. Пусть $\psi$ - характер Гекке. $L$-функцией Гекке назовем ряд Дирихле

$$
L(s, \psi)=\sum_{\mathfrak{a}} \psi(\mathfrak{a}) N(\mathfrak{a})^{-s}, \quad \operatorname{Re} s>1,
$$

где суммирование ведется по целым ненулевым идеалам $\mathfrak{a}$ кольца $\mathbb{Z}_{K}, N(\mathfrak{a})$ - норма идеала $\mathfrak{a}$. Наш подкласс класса Сельберга состоит из $L$-функций Гекке мнимых квадратичных полей.

$L$-функции Гекке, соответствующие неглавным характерам Гекке, аналитически продолжаются на всю комплексную плоскость и удовлетворяют функциональному уравнению вида

$$
L(s, \psi)=\rho(s, \psi) L(1-s, \psi), \quad \rho(s, \psi)=\frac{1}{\theta_{\psi}}\left(\frac{\sqrt{\delta_{K}}}{2 \pi}\right)^{1-2 s} \frac{\Gamma(1-s)}{\Gamma(s)},
$$

где $\delta_{K}$ - дискриминант поля $K, \theta_{\psi}$ - комплексная константа, зависящая только от $\psi$, модуль которой равен 1 (см., например, [10; гл. 7]).

Определим функции $\theta(t, \psi)$ и $Z(t, \psi)$ :

$$
\begin{aligned}
& \exp (i \theta(t, \psi))=\left(\rho\left(\frac{1}{2}+i t\right)\right)^{-1 / 2}, \\
& Z(t, \psi)=\exp (i \theta(t, \psi)) L\left(\frac{1}{2}+i t, \psi\right) .
\end{aligned}
$$

Легко видеть, что при вещественных $t$ функции $\theta(t, \psi)$ и $Z(t, \psi)$ принимают вещественные значения.

В 1997 г. Гриценко [11] доказал неравенство (4) безусловно в случае, когда $F_{1}(s), \ldots, F_{N}(s)$ - $L$-функции Гекке, отвечающие комплексным характерам Гекке одного и того же мнимого квадратичного поля. 
В 2010 г. Резвякова [12] применила к последней задаче метод А. А. Карацубы [4] и получила оценку

$$
N_{0}(T, f) \gg T(\log T)^{2 / h(-D)} \exp \{-c \sqrt{\log \log T}\},
$$

где $h(-D)$ - порядок группы классов идеалов, $c>0$.

В настоящей статье решается задача о нулях функции $f(t)$, определяемой равенствами (3), (6), лежащих на коротких промежутках.

Для того чтобы свести к минимуму трудности, доставляемые арифметикой квадратичного поля, мы ограничиваемся частным случаем, когда $F_{1}(s), \ldots, F_{N}(s)-L$-функции Гекке, отвечающие комплексным характерам Гекке одного и того же мнимого квадратичного поля вида $K=\mathbb{Q}\left(\sqrt{-p_{0}}\right)$, где $p_{0}-$ простое число, сравнимое с 3 по модулю 4.

Отметим, что поля $\mathbb{Q}\left(\sqrt{-p_{0}}\right)$ многоклассны при $p_{0}$ больших некоторой константы. Таблица чисел классов идеалов для некоторых из них приводится в монографии [13].

Сформулируем основной результат статьи.

Теорема 1. Пусть $\varepsilon>0-$ произвольно малое число, $T^{15 / 16+5 \varepsilon} \leqslant H \leqslant T$.

Пусть $F_{1}(s), \ldots, F_{N}(s)$ - L-функиии Гекке, отвечающие комплексным характерам Гекке одного и того же мнимого квадратичного поля вида $\mathbb{Q}\left(\sqrt{-p_{0}}\right)$, где $p_{0}$ - простое число, сравнимое с 3 по модулю 4, а функиия $f(t)$ определена равенством $(3)$, в котором $a_{1}, a_{2}, \ldots, a_{N}-$ произвольные вещественные числа.

Пусть $N_{0}(T, f)$ - число нулей функиии $f(t)$ на отрезке $[0, T]$.

Тогда существует $c>0$ такое, что

$$
N_{0}(T+H, f)-N_{0}(T, f) \gg H(\ln T)^{2 / h} e^{-c \sqrt{\ln \ln T}},
$$

где $h\left(p_{0}\right)$ - число классов идеалов поля $\mathbb{Q}\left(\sqrt{-p_{0}}\right)$.

В дальнейшем будем пользоваться следующими обозначениями:

- $p_{0}$ - простое число, сравнимое с 3 по модулю 4;

- $K=\mathbb{Q}\left(\sqrt{-p_{0}}\right)$ - мнимое квадратичное поле, $\mathbb{Z}_{K}$ - соответствующее кольцо целых алгебраических чисел;

- $\delta_{K}=-p_{0}$ - дискриминант поля $K$;

- $h\left(\delta_{K}\right)$ - число классов идеалов поля $K$;

- $L(s, \psi)-L$-функция Гекке,

$$
L(s, \psi)=\sum_{n=1}^{\infty} a(n) n^{-s}, \quad \operatorname{Re} s>1 ;
$$

- $\bar{n}=\left(\begin{array}{l}n_{1} \\ n_{2}\end{array}\right) \in \mathbb{Z}^{2}$

- $A, A_{1}, A_{2}$ - симметричные матрицы размера $2 \times 2$ с целыми коэффициентами;

- $Q(\bar{n}), Q_{1}(\bar{n}), Q_{2}(\bar{n})$ - бинарные примитивные квадратичные формы дискриминанта $\delta_{K}$, связанные с матрицами $A, A_{1}, A_{2}$ формулами

$$
Q(\bar{n})=\frac{1}{2} \bar{n}^{t} A \bar{n}, \quad Q_{1}(\bar{n})=\frac{1}{2} \bar{n}^{t} A_{1} \bar{n}, \quad Q_{2}(\bar{n})=\frac{1}{2} \bar{n}^{t} A_{2} \bar{n} ;
$$

- $Q^{\prime}(\bar{n}), Q_{1}^{\prime}(\bar{n}), Q_{2}^{\prime}(\bar{n})$ - квадратичные формы с матрицами $p_{0} A^{-1}, p_{0} A_{1}^{-1}, p_{0} A_{2}^{-1}$;

- $G(q, u, \bar{m})$ - сумма Гаусса, отвечающая квадратичной форме $Q(\bar{n})$,

$$
G(q, u, \bar{m})=\sum_{\bar{g}(\bmod q)} \exp \left(2 \pi i \frac{u Q(\bar{g})+\bar{m}^{t} \bar{g}}{q}\right),
$$

$G_{1}(q, u, \bar{m}), G_{2}(q, u, \bar{m})$ - суммы Гаусса, отвечающие квадратичным формам $Q_{1}(\bar{n})$, $Q_{2}(\bar{n})$. 


\section{2. Вспомогательные утверждения}

В методе А. А. Карацубы [6] важную роль играют приближенные функциональные уравнения рядов Дирихле. Для $L$-функций Гекке известно, например, приближенное функциональное уравнение Лаврика [14]. Однако наличие в его формуле остаточного члена, который при $s=1 / 2+i t$ может расти как $\log ^{2}|t|$, когда $|t| \rightarrow \infty$, не дает возможности применить ее к нашей задаче. Мы выведем приближенное функциональное уравнение $L$-функции Гекке несколько иного вида, чем у Лаврика. Его преимущество состоит в малости остаточного члена.

Лемма 1. Пусть $\varepsilon>0$ - произвольно малое число, $\psi$ - комплексный характер Гекке,

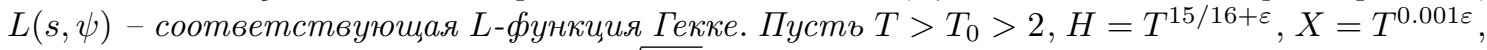
$T-1 \leqslant t \leqslant T+H+1, P=P(t)=t \sqrt{\left|\delta_{K}\right|} / 2 \pi$. Пусть $\nu_{1}$ и $\nu_{2}-$ натуральнье числа, меньuиe $X, s=1 / 2+i t$.

Тогда справедливо равенство

$$
L(s, \psi)=\sum_{n=1}^{\infty} a(n) n^{-s} \exp \left(-\frac{n \nu_{1}}{P \nu_{2}}\right)+\rho(s, \psi) \sum_{n=1}^{\infty} a(n) n^{s-1}\left(1-\exp \left(-\frac{P \nu_{2}}{n \nu_{1}}\right)\right)+O\left(T^{(\varepsilon-1) / 2}\right) .
$$

ДоказАтЕльство. Начнем с равенства Меллина

$$
\exp \left(-\frac{n \nu_{1}}{P \nu_{2}}\right)=\frac{1}{2 \pi i} \int_{2-i \infty}^{2+i \infty} \Gamma(w)\left(\frac{P \nu_{2}}{n \nu_{1}}\right)^{w} d w .
$$

Умножим обе его части на $a(n) n^{-s}$ и просуммируем получившееся равенство по $n$ от 1 до $\infty$ :

$$
\sum_{n=1}^{\infty} a(n) n^{-s} \exp \left(-\frac{n \nu_{1}}{P \nu_{2}}\right)=\frac{1}{2 \pi i} \int_{2-i \infty}^{2+i \infty} \Gamma(w)\left(\frac{P \nu_{2}}{\nu_{1}}\right)^{w} L(s+w, \psi) d w .
$$

Будем преобразовывать интеграл, стоящий в правой части, который обозначим через $I$. Пользуясь следствием из формулы Стирлинга

$$
\Gamma(2+i v) \ll(2+|v|)^{3 / 2} e^{-\pi|v| / 2},
$$

получаем

$$
I=\frac{1}{2 \pi i} \int_{2-i \log ^{2} T}^{2+i \log ^{2} T} \Gamma(w)\left(\frac{P \nu_{2}}{\nu_{1}}\right)^{w} L(s+w, \psi) d w+O\left(T^{-1}\right) .
$$

Пользуясь неравенством

$$
L(\sigma+i t, \psi)=O\left((|t|+2)^{1-\sigma} \log ^{3}(|t|+2)\right),
$$

выполняющимся при $-1 / \log (|t|+2) \leqslant \sigma \leqslant 1+\log (|t|+2)$ и прямо следующим из приближенного функционального уравнения Лаврика, перенесем отрезок интегрирования на

$$
\operatorname{Re} w=-\beta_{0}, \quad-\log ^{2} T \leqslant \operatorname{Im} w \leqslant \log ^{2} T,
$$

где $\beta_{0}=1 / 2+1 / 2 \log T$. При этом "пройдем" через простой полюс в точке $w=0$ с вычетом $L(s, \psi)$; по теореме Коши имеем

$$
I=L(s, \psi)+I_{1}+O\left(T^{-1}\right),
$$

где

$$
I_{1}=\frac{1}{2 \pi i} \int_{-\beta_{0}-i \log ^{2} T}^{-\beta_{0}+i \log ^{2} T} \Gamma(w)\left(\frac{P \nu_{2}}{\nu_{1}}\right)^{w} L(s+w, \psi) d w .
$$


Воспользуемся функциональным уравнением $L$-функции Гекке

$$
L(s+w, \psi)=\rho(s+w, \psi) L(1-s-w, \psi),
$$

где

$$
\rho(s+w, \psi)=\frac{1}{\theta_{\psi}}\left(\frac{\delta_{K}}{2 \pi}\right)^{1-2 s-2 w} \frac{\Gamma(1-s-w)}{\Gamma(s+w)} .
$$

Из формулы Стирлинга следует, что

$$
\rho(s+w, \psi)=\rho(s, \psi) P^{-2 w}\left(1+O\left(T^{-1}\right)+O\left(|w|^{2} T^{-1}\right)\right) .
$$

Отсюда имеем

$$
I_{1}=\rho(s, \psi) \frac{1}{2 \pi i} \int_{-\beta_{0}-i \log ^{2} T}^{-\beta_{0}+i \log ^{2} T} \Gamma(w)\left(\frac{\nu_{2}}{P \nu_{1}}\right)^{w} L(s+w, \psi) d w+O\left(T^{(\varepsilon-1) / 2}\right) .
$$

Далее, распространяем отрезок интегрирования на всю прямую $\operatorname{Re} w=-\beta_{0}$, представляем $L(1-s-w, \psi)$ в виде суммы ряда Дирихле (учитывая неравенство $\operatorname{Re}(1-s-w)>1$ ) и меняем порядок суммирования и интегрирования:

$$
I_{1}=\rho(s, \psi) \sum_{n=1}^{\infty} a(n) n^{s-1} \frac{1}{2 \pi i} \int_{-\beta_{0}-i \infty}^{-\beta_{0}+i \infty} \Gamma(w)\left(\frac{n \nu_{2}}{P \nu_{1}}\right)^{w} d w+O\left(T^{(\varepsilon-1) / 2}\right) .
$$

Теперь утверждение леммы следует из равенства

$$
\frac{1}{2 \pi i} \int_{-\beta_{0}-i \infty}^{-\beta_{0}+i \infty} \Gamma(w)\left(\frac{n \nu_{2}}{P \nu_{1}}\right)^{w} d w=-1+\exp \left(-\frac{P \nu_{2}}{n \nu_{1}}\right) .
$$

СлеДСтвиЕ 1. Пусть $|u| \leqslant 1$. Тогда справедливо равенство

$$
\begin{aligned}
Z(t+u, \psi)=\theta_{\psi}^{1 / 2} & \sum_{n=1}^{\infty} \frac{a(n)}{\sqrt{n}}\left(\frac{P}{n}\right)^{i(t+u)} e^{-i t} \exp \left(-\frac{n \nu_{1}}{P \nu_{2}}\right) \\
& +\theta_{\psi}^{-1 / 2} \sum_{n=1}^{\infty} \frac{a(n)}{\sqrt{n}}\left(\frac{P}{n}\right)^{-i(t+u)} e^{i t}\left(1-\exp \left(-\frac{P \nu_{2}}{n \nu_{1}}\right)\right)+O\left(T^{(2 \varepsilon-1) / 2}\right) .
\end{aligned}
$$

ДокАЗАТЕЛЬСтво. Положим в равенстве леммы $1 t+u$ вместо $t$, воспользуемся формулой Стирлинга и неравенством $|a(n)| \leqslant \tau(n)$ (см., например, [11]).

Следствие 1 представляет собой приближенное функциональное уравнение для аналога функции Харди. Нам потребуется приближенное функциональное уравнение для аналога функции, названной А. А. Карацубой функцией Харди-Сельберга.

Пусть $\beta(\nu)$ - вещественные числа, не превосходящие $\tau(\nu)$, значения которых будут уточнены позже. Пусть $\phi_{X}(s)=\sum_{\nu<X} \beta(\nu) \nu^{-s}$, где $X$ - параметр, определенный в лемме 1 . Пусть

$$
F(t, \psi)=Z(t, \psi)\left|\phi_{X}\left(\frac{1}{2}+i t\right)\right|^{2}
$$

ЛЕмма 2. В условиях леммы 1 справедливо равенство

$$
\begin{aligned}
F(t+u, \psi)=\theta_{\psi}^{1 / 2} & \sum_{\lambda} \frac{A(\lambda)}{\sqrt{\lambda}}\left(\frac{P}{\lambda}\right)^{i(t+u)} e^{-i t} \exp \left(-\frac{\lambda}{P}\right) \\
& +\theta_{\psi}^{-1 / 2} \sum_{\lambda} \frac{A(\lambda)}{\sqrt{\lambda}}\left(\frac{P}{\lambda}\right)^{-i(t+u)} e^{i t}\left(1-\exp \left(-\frac{P}{\lambda}\right)\right)+O\left(T^{(3 \varepsilon-1) / 2}\right),
\end{aligned}
$$


где

$$
A(\lambda)=\sum_{n \nu_{1} / \nu_{2}=\lambda} \frac{a(n) \beta\left(\nu_{1}\right) \beta\left(\nu_{2}\right)}{\nu_{2}}
$$

(суммирование ведется по натуральным $\nu_{1} u \nu_{2}$, менъшим $X$, и произвольным натуральнзмм $n$ ).

ДокАзАтЕльство. Умножим обе части равенства из следствия 1 на

$$
\frac{\beta\left(\nu_{1}\right) \beta\left(\nu_{2}\right)}{\sqrt{\nu_{1} \nu_{2}}}\left(\frac{\nu_{1}}{\nu_{2}}\right)^{-i(t+u)}
$$

и просуммируем получившееся равенство по натуральным $\nu_{1}$ и $\nu_{2}$, меньшим $X$.

В следствии 2 формула леммы 2 приводится к виду, более удобному для приложений.

СлеДСтвиЕ 2. Пусть $P_{0}=T \sqrt{\left|\delta_{K}\right|} / 2 \pi$. В условиях леммы 2 справедливо равенство

$$
\begin{aligned}
& F(t+u, \psi)=\theta_{\psi}^{1 / 2} W_{1}(t+u, \psi)+\theta_{\psi}^{-1 / 2} W_{2}(t+u, \psi)+\theta_{\psi}^{-1 / 2} W_{3}(t+u, \psi) \\
& +\theta_{\psi}^{-1 / 2} \sum_{\nu=1}^{\infty} W_{4}(t+u, \psi, \nu)+\theta_{\psi}^{1 / 2} \sum_{\substack{\mu=0 \\
\mu+\nu>0}}^{\infty} \sum_{\nu=0}^{\infty} W_{1}(t+u, \psi, \mu, \nu) \\
& +\theta_{\psi}^{-1 / 2} \sum_{\mu=1}^{\infty} W_{2}(t+u, \psi, \mu)+\theta_{\psi}^{-1 / 2} \sum_{\substack{\mu=0 \\
\mu+\nu>0}}^{\infty} \sum_{\substack{\nu=0 \\
\infty}}^{\infty} W_{3}(t+u, \psi, \mu, \nu) \\
& +\theta_{\psi}^{-1 / 2} \sum_{\mu=1}^{\infty} \sum_{\nu=1}^{\infty} W_{4}(t+u, \psi, \mu, \nu)+O\left(T^{(3 \varepsilon-1) / 2}\right),
\end{aligned}
$$

где

$$
\begin{aligned}
W_{1}(t+u, \psi) & =\sum_{\lambda \leqslant P_{0} \log ^{2} T} \frac{A(\lambda)}{\sqrt{\lambda}}\left(\frac{P}{\lambda}\right)^{i t}\left(\frac{P_{0}}{\lambda}\right)^{i u} e^{-i t} \exp \left(-\frac{\lambda}{P_{0}}\right), \\
W_{2}(t+u, \psi) & =\sum_{\lambda \leqslant P_{0}} \frac{A(\lambda)}{\sqrt{\lambda}}\left(\frac{P}{\lambda}\right)^{-i t}\left(\frac{P_{0}}{\lambda}\right)^{-i u} e^{i t}, \\
W_{3}(t+u, \psi) & =\sum_{P_{0} \log ^{-2} T<\lambda \leqslant P_{0}} \frac{A(\lambda)}{\sqrt{\lambda}}\left(\frac{P}{\lambda}\right)^{-i t}\left(\frac{P_{0}}{\lambda}\right)^{-i u} e^{i t} \exp \left(-\frac{P_{0}}{\lambda}\right), \\
W_{4}(t+u, \psi, \nu) & =\sum_{P_{0}<\lambda} \frac{A(\lambda)}{\sqrt{\lambda}}\left(\frac{P}{\lambda}\right)^{-i t}\left(\frac{P_{0}}{\lambda}\right)^{-i u} e^{i t} \frac{(-1)^{\nu}}{\nu !}\left(\frac{P}{\lambda}\right)^{\nu}, \\
W_{1}(t+u, \psi, \mu, \nu) & =\sum_{\lambda \leqslant P_{0} \log ^{2} T} \frac{A(\lambda)}{\sqrt{\lambda}}\left(\frac{P}{\lambda}\right)^{i t}\left(\frac{P_{0}}{\lambda}\right)^{i u} e^{-i t} \exp \left(-\frac{\lambda}{P_{0}}\right)^{\nu} \\
W_{2}(t+u, \psi, \mu) & =\sum_{\lambda \leqslant P_{0}} \frac{\frac{A(\lambda)}{\nu !}\left(\frac{P u}{\lambda}\right)^{-i t}\left(\frac{P_{0}}{P_{0}}\right)^{\nu}\left(\frac{P-P_{0}}{P_{0}}\right)^{\nu}\left(\frac{P-P_{0}}{P}\right)^{\mu},}{e^{i t}\left(\begin{array}{c}
-i u \\
\mu
\end{array}\right)\left(\frac{P-P_{0}}{P}\right)^{\mu},}
\end{aligned}
$$




$$
\begin{gathered}
W_{3}(t+u, \psi, \mu, \nu)=\sum_{P_{0} \log ^{-2} T<\lambda \leqslant P_{0}} \frac{A(\lambda)}{\sqrt{\lambda}}\left(\frac{P}{\lambda}\right)^{-i t}\left(\frac{P_{0}}{\lambda}\right)^{-i u} e^{i t} \exp \left(-\frac{P_{0}}{\lambda}\right) \\
\times \frac{1}{\nu !}\left(\begin{array}{c}
-i u \\
\mu
\end{array}\right)\left(\frac{P-P_{0}}{P}\right)^{\mu}\left(\frac{P-P_{0}}{\lambda}\right)^{\nu}, \\
W_{4}(t+u, \psi, \mu, \nu)=\sum_{P_{0}<\lambda} \frac{A(\lambda)}{\sqrt{\lambda}}\left(\frac{P}{\lambda}\right)^{-i t}\left(\frac{P_{0}}{\lambda}\right)^{-i u} e^{i t} \frac{(-1)^{\nu}}{\nu !}\left(\frac{P}{\lambda}\right)^{\nu}\left(\begin{array}{c}
-i u \\
\mu
\end{array}\right)\left(\frac{P-P_{0}}{P_{0}}\right)^{\mu} .
\end{gathered}
$$

ДокАЗАТЕЛьство. Из леммы 2 имеем

$$
\begin{aligned}
F(t+u, \psi)=\theta_{\psi}^{1 / 2} & \sum_{\lambda \leqslant P_{0} \log ^{2} T} \frac{A(\lambda)}{\sqrt{\lambda}}\left(\frac{P}{\lambda}\right)^{i(t+u)} e^{-i t} \exp \left(-\frac{\lambda}{P}\right)+\theta_{\psi}^{-1 / 2} \sum_{\lambda \leqslant P_{0}} \frac{A(\lambda)}{\sqrt{\lambda}}\left(\frac{P}{\lambda}\right)^{-i(t+u)} e^{i t} \\
& +\theta_{\psi}^{-1 / 2} \sum_{P_{0} \log ^{-2} T<\lambda \leqslant P_{0}} \frac{A(\lambda)}{\sqrt{\lambda}}\left(\frac{P}{\lambda}\right)^{-i(t+u)} e^{i t} \exp \left(-\frac{P}{\lambda}\right) \\
& +\theta_{\psi}^{-1 / 2} \sum_{P_{0}<\lambda} \frac{A(\lambda)}{\sqrt{\lambda}}\left(\frac{P}{\lambda}\right)^{-i(t+u)} e^{i t}\left(1-\exp \left(-\frac{P}{\lambda}\right)\right)+O\left(T^{(3 \varepsilon-1) / 2}\right) .
\end{aligned}
$$

Теперь утверждение следствия прямо следует из равенств

$$
\begin{aligned}
P^{ \pm i u} & =P_{0}^{ \pm i u}\left(1+\frac{P-P_{0}}{P_{0}}\right)^{ \pm i u}=P_{0}^{ \pm i u} \sum_{\mu=0}^{\infty}\left(\begin{array}{c} 
\pm i u \\
\mu
\end{array}\right)\left(\frac{P-P_{0}}{P_{0}}\right)^{\mu}, \\
\exp \left(-\frac{\lambda}{P}\right) & =\exp \left(-\frac{\lambda}{P_{0}}\right) \exp \left(\frac{\lambda}{P_{0}} \frac{P-P_{0}}{P}\right) \\
& =\exp \left(-\frac{\lambda}{P_{0}}\right) \sum_{\nu=0}^{\infty} \frac{1}{\nu !}\left(\frac{\lambda}{P_{0}}\right)^{\nu}\left(\frac{P-P_{0}}{P}\right)^{\nu} \quad\left(\lambda \leqslant P_{0} \log ^{2} T\right), \\
1 & -\exp \left(-\frac{P}{\lambda}\right)=\sum_{\nu=1}^{\infty} \frac{(-1)^{\nu-1}}{\nu !}\left(\frac{P}{\lambda}\right)^{\nu} \quad\left(P_{0}<\lambda\right) .
\end{aligned}
$$

\section{3. Аддитивная задача}

Лемма 3. Пусть $\operatorname{Im} \tau>0, \bar{x} \in \mathbb{R}^{2}, Q(\bar{n})$ - положительно определенная квадратичная борма дискриминанта $\delta_{F}$ с матрицей $A$,

$$
\theta_{Q}(\tau, \bar{x})=\sum_{\bar{n} \in \mathbb{Z}^{2}} e^{2 \pi i \tau Q(\bar{n}+\bar{x})} .
$$

Тогда справедливо тождество

$$
\theta_{Q}(\tau, \bar{x})=\frac{i}{\tau \sqrt{\left|\delta_{F}\right|}} \sum_{\bar{m} \in \mathbb{Z}^{2}} \exp \left(-\frac{\pi i}{\tau} \bar{m}^{t} A^{-1} \bar{m}+2 \pi i \bar{m}^{t} \bar{x}\right) .
$$

ДоказАтельство см., например, в [15; гл. 7].

СлеДСтвиЕ 3. Пусть $\operatorname{Im} \tau>0, \bar{x} \in \mathbb{R}^{2}, \bar{y} \in \mathbb{R}^{2}, Q(\bar{n}), Q^{\prime}(\bar{n})$ - положительно определенные квадратичные формы с матрицами $A$ и $p_{0} A^{-1}$ соответственно, $\operatorname{det} A=p_{0}$.

Тогда справедливо тождество

$$
\sum_{\bar{n} \in \mathbb{Z}^{2}} \exp \left\{2 \pi i \tau Q(\bar{n}+\bar{x})+2 \pi i \bar{y}^{t}(\bar{n}+\bar{x})\right\}=\frac{i}{\tau \sqrt{p_{0}}} \sum_{\bar{n} \in \mathbb{Z}^{2}} \exp \left\{-\frac{2 \pi i}{\tau p_{0}} Q^{\prime}(\bar{n}-\bar{y})+2 \pi i \bar{x}^{t} \bar{n}\right\} .
$$


ДокАЗАТЕЛЬСтво. По определению имеем

$$
Q^{\prime}(\bar{n}-\bar{y})=(\bar{n}-\bar{y})^{t} \frac{1}{2} p_{0} A^{-1}(\bar{n}-\bar{y})=Q^{\prime}(\bar{n})+Q^{\prime}(\bar{y})-p_{0} \bar{y}^{t} A^{-1} \bar{n},
$$

поэтому

$$
\begin{aligned}
& \frac{i}{\tau \sqrt{p_{0}}} \sum_{\bar{n} \in \mathbb{Z}^{2}} \exp \left\{-\frac{2 \pi i}{\tau p_{0}} Q^{\prime}(\bar{n}-\bar{y})+2 \pi i \bar{x}^{t} \bar{n}\right\} \\
& \quad=\exp \left\{-\frac{2 \pi i}{\tau p_{0}} Q^{\prime}(\bar{y})\right\} \frac{i}{\tau \sqrt{p_{0}}} \sum_{\bar{n} \in \mathbb{Z}^{2}} \exp \left\{-\frac{2 \pi i}{\tau p_{0}} Q^{\prime}(\bar{n})+2 \pi i\left(\bar{x}^{t}+\frac{\bar{y}^{t} A^{-1}}{\tau}\right) \bar{n}\right\} .
\end{aligned}
$$

Воспользуемся леммой 3:

$$
\begin{aligned}
& \frac{i}{\tau \sqrt{p_{0}}} \sum_{\bar{n} \in \mathbb{Z}^{2}} \exp \left\{-\frac{2 \pi i}{\tau p_{0}} Q^{\prime}(\bar{n}-\bar{y})+2 \pi i \bar{x}^{t} \bar{n}\right\} \\
& \quad=\exp \left\{-\frac{2 \pi i}{\tau p_{0}} Q^{\prime}(\bar{y})\right\} \sum_{\bar{n} \in \mathbb{Z}^{2}} \exp \left\{2 \pi i \tau Q\left(\bar{n}+\bar{x}+\frac{A^{-1} \bar{y}}{\tau}\right)\right\} .
\end{aligned}
$$

Далее,

$$
Q\left(\bar{n}+\bar{x}+\frac{A^{-1} \bar{y}}{\tau}\right)=Q(\bar{n}+\bar{x})+\frac{Q^{\prime}(\bar{y})}{\tau^{2} p_{0}}+\frac{1}{\tau} \bar{y}^{t}(\bar{n}+\bar{x})
$$

поэтому

$$
\frac{i}{\tau \sqrt{p_{0}}} \sum_{\bar{n} \in \mathbb{Z}^{2}} \exp \left\{-\frac{2 \pi i}{\tau p_{0}} Q^{\prime}(\bar{n}-\bar{y})+2 \pi i \bar{x}^{t} \bar{n}\right\}=\sum_{\bar{n} \in \mathbb{Z}^{2}} \exp \left\{2 \pi i \tau Q(\bar{n}+\bar{x})+2 \pi i \bar{y}^{t}(\bar{n}+\bar{x})\right\} .
$$

Следствие доказано.

Лемма 4. Пусть $\varepsilon>0$ - сколь угодно малое число, а и $b$-натуральные взаимно простые числа, $1 \leqslant a, b \leqslant y^{\varepsilon}, k<y$ - натуральное число. Пусть $\psi$ - комплексный характер группы классов идеалов кольиа $\mathbb{Z}_{K}$,

$$
J_{k}(y, a, b)=\sum_{\substack{\mathfrak{a}, \mathfrak{b} \\ a N(\mathfrak{a})-b N(\mathfrak{b})=k}} \psi(\mathfrak{a}) \bar{\psi}(\mathfrak{b}) e^{-(a N(\mathfrak{a})+b N(\mathfrak{b})) / y},
$$

где суммирование ведется по целым ненулевым идеалам кольца $\mathbb{Z}_{F}$.

Тогда справедлива ощенка $J_{k}(y, a, b)=O\left(y^{3 / 4+5 \varepsilon}\right)$.

ДокаЗАТЕЛЬСТво см. в [16].

Пусть $X_{2}-X_{1}>1$. Определим функцию $g\left(x, X_{1}, X_{2}\right)$ :

$$
g\left(x, X_{1}, X_{2}\right)= \begin{cases}0, & \text { если } x \leqslant X_{1} \text { или } x \geqslant X_{2}+1, \\ 1, & \text { если } X_{1}+1 \leqslant x \leqslant X_{2}, \\ x-X_{1}, & \text { если } X_{1} \leqslant x \leqslant X_{1}+1, \\ X_{2}+1-x, & \text { если } X_{2} \leqslant x \leqslant X_{2}+1 .\end{cases}
$$

ЛЕмма 5 (функциональное уравнение неполной тэта-функции). Пусть $Y>10, x \in \mathbb{R}$, $l \in \mathbb{Z}, q \in \mathbb{N}, b \in \mathbb{Z}$,

$$
\tau=\left(-\frac{l}{q}-x+\frac{i}{2 \pi Y}\right) b q^{2} .
$$

Пусть $N_{1}, N_{2}, N_{3}, N_{4}$ - челье числа, $P$ - прямоугольник вида $\left(N_{1}, N_{2}\right] \times\left(N_{3}, N_{4}\right]$. 
Тогда справедливо тождество

$$
\begin{aligned}
\sum_{\bar{n} \in P} \exp \left(2 \pi i\left(-\frac{l}{q}-x+\frac{i}{2 \pi Y}\right) b Q_{2}(\bar{n})\right) \\
=\frac{i}{2 \pi \sqrt{p_{0}} \tau} \int_{-\infty}^{+\infty} \int_{-\infty}^{+\infty} \widehat{g}\left(v_{1}, N_{1}, N_{2}\right) \widehat{g}\left(v_{2}, N_{3}, N_{4}\right) \\
\quad \times \sum_{\bar{n} \in \mathbb{Z}^{2}} \exp \left(-\frac{2 \pi i}{p_{0} \tau} Q_{2}\left(\bar{n}-\frac{q \bar{v}}{2 \pi}\right)\right) G_{2}(q,-b l, \bar{n}) d v_{1} d v_{2} .
\end{aligned}
$$

ДокАЗАТЕЛЬСТво. Имеем тождество

$$
\begin{aligned}
\sum_{\bar{n} \in P} \exp \left(-2 \pi i\left(\frac{l}{q}-x+\frac{i}{2 \pi Y}\right) b Q_{2}(\bar{n})\right) \\
\quad=\sum_{n_{1}=-\infty}^{\infty} \sum_{n_{1}=-\infty}^{\infty} g\left(n_{1}, N_{1}, N_{2}\right) g\left(n_{2}, N_{3}, N_{4}\right) \exp \left(2 \pi i\left(-\frac{l}{q}-x+\frac{i}{2 \pi Y}\right) b Q_{2}(\bar{n})\right) .
\end{aligned}
$$

Выразим функцию $g\left(x, X_{1}, X_{2}\right)$ через ее преобразование Фурье:

$$
\begin{aligned}
& \sum_{n_{1}=-\infty}^{\infty} \sum_{n_{1}=-\infty}^{\infty} g\left(n_{1}, N_{1}, N_{2}\right) g\left(n_{2}, N_{3}, N_{4}\right) \exp \left(2 \pi i\left(-\frac{l}{q}-x+\frac{i}{2 \pi Y}\right) b Q_{2}\left(n_{1}, n_{2}\right)\right) \\
& =\frac{1}{2 \pi} \int_{-\infty}^{+\infty} \int_{-\infty}^{+\infty} \widehat{g}\left(v_{1}, N_{1}, N_{2}\right) \widehat{g}\left(v_{2}, N_{3}, N_{4}\right) \\
& \quad \times \sum_{\bar{n} \in \mathbb{Z}^{2}} \exp \left(2 \pi i\left(-\frac{l}{q}-x+\frac{i}{2 \pi Y}\right) b Q_{2}(\bar{n})\right) \exp \left(2 \pi i \frac{\bar{v}^{t} \bar{n}}{2 \pi}\right) d v_{1} d v_{2},
\end{aligned}
$$

где $\bar{n}=\left(\begin{array}{l}n_{1} \\ n_{2}\end{array}\right), \bar{v}=\left(\begin{array}{l}v_{1} \\ v_{2}\end{array}\right)$.

Последнюю сумму разобьем по арифметической прогрессии с разностью $q$ :

$$
\begin{aligned}
& \sum_{\bar{n} \in \mathbb{Z}^{2}} \exp \left(2 \pi i\left(-\frac{l}{q}-x+\frac{i}{2 \pi Y}\right) b Q_{2}(\bar{n})\right) \exp \left(2 \pi i \frac{\bar{v}^{t} \bar{n}}{2 \pi}\right) d v_{1} d v_{2} \\
& \quad=\sum_{\bar{s} \bmod q} \exp \left(-2 \pi i \frac{l b Q_{2}(\bar{s})}{q}\right) \sum_{\bar{n} \in \mathbb{Z}^{2}} \exp \left(2 \pi i \tau Q_{2}\left(\bar{n}+\frac{\bar{s}}{q}\right)\right) \exp \left(2 \pi i \frac{q \bar{v}^{t}(\bar{n}+\bar{s} / q)}{2 \pi}\right) .
\end{aligned}
$$

Воспользуемся следствием 3:

$$
\begin{aligned}
& \sum_{\bar{n} \in \mathbb{Z}^{2}} \exp \left(2 \pi i \tau Q_{2}\left(\bar{n}+\frac{\bar{s}}{q}\right)\right) \exp \left(2 \pi i \frac{q \bar{v}^{t}(\bar{n}+\bar{s} / q)}{2 \pi}\right) \\
& =\frac{i}{\tau \sqrt{p_{0}}} \sum_{\bar{n} \in \mathbb{Z}^{2}} \exp \left(-\frac{2 \pi i}{\tau p_{0}} Q_{2}\left(\bar{n}-\frac{q \bar{v}}{2 \pi}\right)\right) \exp \left(\frac{2 \pi i \bar{n}^{t} \bar{s}}{q}\right) .
\end{aligned}
$$

Утверждение прямо следует из приведенных выше тождеств.

ЛЕмма 6. Пусть $\varepsilon>0$ - сколь угодно малое число, у $>10, a$ u $b$ - натуральные числа, $1 \leqslant a, b \leqslant y^{\varepsilon},(a, b)=1, k$ - натуральное число, меньшее $y$,

$$
W(y, a, b, k)=\sum_{\substack{a m-b n=k \\ m \leqslant y}} a(m) a(n) .
$$

Тогда справедлива ощенка

$$
W(y, a, b, k) \ll y^{7 / 8+6 \varepsilon} .
$$


ДокАЗАтЕльство. Пусть $Y=y^{1+\varepsilon}$. Определим

$$
a\left(\frac{b n+k}{a}\right)=0 \quad \text { при } \quad \frac{b n+k}{a} \notin \mathbb{N} .
$$

Пользуясь преобразованием Абеля, имеем

$$
\begin{aligned}
\sum_{\substack{a m-b n=k, n \leqslant y}} a(m) a(n)= & \sum_{m \leqslant y} a\left(\frac{b n+k}{a}\right) a(n) \exp \left(-\frac{2 b n+k}{Y}\right) \exp \left(\frac{2 b n+k}{Y}\right) \\
= & -\int_{1}^{y}\left(\sum_{\substack{a m-b n=k, m \leqslant R}} a(m) a(n) \exp \left(-\frac{a m+b n}{Y}\right)\right) d \exp \left(\frac{2 b R+k}{Y}\right) \\
& +\sum_{\substack{a m-b n=k, m \leqslant y}} a(m) a(n) \exp \left(-\frac{a m+b n}{Y}\right) \exp \left(\frac{2 b y+k}{Y}\right) .
\end{aligned}
$$

Теперь достаточно оценить сумму

$$
\sum_{\substack{a m-b n=k, m \leqslant R}} a(m) a(n) \exp \left(-\frac{a m+b n}{Y}\right)
$$

при $R \leqslant y$.

Как и при доказательстве леммы 4, сводим задачу к вычислению асимптотики суммы

$$
I_{k}(R, a, b, \mathcal{A}, \mathcal{B})=\sum_{\substack{a Q_{1}(\bar{m})-b Q_{2}(\bar{n})=k \\ Q_{2}(\bar{n}) \leqslant R}} \exp \left(-\frac{a Q_{1}(\bar{m})+b Q_{2}(\bar{n})}{Y}\right),
$$

где $Q_{1}(\bar{m})$ и $Q_{2}(\bar{n})$ - те положительно определенные бинарные квадратичные формы, которые представляют нормы идеалов из классов $\mathcal{A}$ и $\mathcal{B}$ соответственно.

Если вместо условия $Q_{2}(\bar{n}) \leqslant R$ мы бы имели условие $Q_{2}(\bar{n}) \in P$, где $P=\left(N_{1}, N_{2}\right] \times\left(N_{3}, N_{4}\right]$, то, по существу повторяя доказательство леммы 4 , пришли бы к оценке

$$
\sum_{\mathcal{A}} \sum_{\mathcal{B}} \psi(\mathcal{A}) \psi(\mathcal{B}) I_{k}(R, a, b, \mathcal{A}, \mathcal{B})=O\left(Y^{3 / 4+6 \varepsilon}\right)
$$

Единственное отличие состоит здесь в том, что одна из тригонометрических сумм, возникающих при применении кругового метода, выражается через неполную тэта-функцию из леммы 5. Применяя к ней преобразование из леммы 5, а затем дословно повторяя рассуждения из доказательства леммы 4 и пользуясь неравенством

$$
\int_{-\infty}^{+\infty}\left|\widehat{g}\left(v, N_{1}, N_{2}\right)\right| d v \ll \log \left(N_{2}-N_{1}+2\right),
$$

приходим к (7).

Если доказать, что внутренность эллипса $Q_{2}(\bar{n}) \leqslant R$ может быть представлена в виде объединения $O\left(Y^{1 / 8}\right)$ прямоугольников со сторонами, параллельными координатным осям, и некоторого количества криволинейных треугольников, общая площадь которых есть $O\left(Y^{7 / 8}\right)$, лемма 6 будет доказана.

Внутренность эллипса можно разбить на конечное число прямоугольников со сторонами, параллельными координатным осям, и криволинейных треугольников. Эти фигуры попарно не пересекаются. 
Без ограничения общности считаем, что криволинейный треугольник имеет вид

$$
\alpha \leqslant m_{1} \leqslant \beta, \quad \omega \leqslant m_{2} \leqslant f\left(m_{1}\right),
$$

где

$$
- \text { const } \cdot \sqrt{R} \leqslant \alpha<\beta \leqslant \text { const } \cdot \sqrt{R}, \quad|\omega| \leqslant \text { const } \cdot \sqrt{R},
$$

a $f(x)$ - монотонно возрастающая дифференцируемая функция, производная которой ограничена на $[\alpha, \beta]$.

Если $\beta-\alpha \leqslant R^{3 / 8}$, то площадь криволинейного треугольника есть $O\left(R^{3 / 4}\right)$.

Пусть $\beta-\alpha>R^{3 / 8}$. Разобьем отрезок $[\alpha, \beta]$ на отрезки длины $R^{3 / 8}$ и рассмотрим нижнюю интегральную сумму Дарбу функции $f(x)$, соответствующую этому разбиению. Нижняя сумма Дарбу равна сумме площадей прямоугольников, число которых не превосходит const. $R^{1 / 8}$. Разность между $\int_{\alpha}^{\beta} f(x) d x$ и нижней суммой Дарбу равна сумме площадей криволинейных треугольников, общая площадь которых не превосходит const $\cdot R^{7 / 8}$.

Тем самым, нужный факт доказан, а вместе с ним доказана и лемма 6.

\section{4. Доказательство основной теоремы}

Следуем схеме рассуждений работы [17], внося в нее необходимые изменения.

Пусть $P_{1}-$ множество простых чисел, являющихся нормами простых главных идеалов, причем $p_{0} \notin P_{1}$.

Хорошо известно, что

$$
\lim _{x \rightarrow \infty}(\pi(x))^{-1} \sum_{\substack{p \leqslant x \\ p \in P_{1}}} 1=\frac{1}{2 h\left(-p_{0}\right)}
$$

(см., например, [18]).

Все функции, сумма которых стоит в правой части (3), имеют общий множитель

$$
\prod_{p \in P_{1}}\left(1-p^{-s}\right)^{-1} .
$$

Определим последовательности $\alpha(\nu)$ и $\beta(\nu)$ :

$$
\begin{gathered}
\sum_{\nu=1}^{\infty} \alpha(\nu) \nu^{-s}=\prod_{p \in P_{1}}\left(1-p^{-s}\right), \quad \operatorname{Re} s>1, \\
\beta(\nu)= \begin{cases}\alpha(\nu)\left(1-\frac{\log \nu}{\log X}\right), & \text { если } 1 \leqslant \nu \leqslant X, \\
0, & \text { если } X<\nu,\end{cases}
\end{gathered}
$$

где $X=T^{0.001 \varepsilon}$.

Положим $\phi_{X}(s)=\sum_{\nu<X} \beta(\nu) \nu^{-s}$. Тогда нули нечетного порядка функций

$$
F(t)=\sum_{j=1}^{N} a_{j} Z\left(t, \psi_{j}\right)\left|\phi_{X}\left(\frac{1}{2}+i t\right)\right|^{2}
$$

являются нулями нечетного порядка функции

$$
\sum_{j=1}^{N} a_{j} Z\left(t, \psi_{j}\right)
$$


Введем параметры $c_{4}, h, h_{1}, \alpha$ :

$$
\begin{gathered}
\frac{1}{c_{4}}=(\log T)^{1-2 / h\left(-p_{0}\right)} \exp (\sqrt{\log \log T}), \quad h=\frac{1}{c_{4} \log T} \sqrt{\log \frac{1}{c_{4}}}, \\
h_{1}=h \sqrt{\log \frac{1}{c_{4}}}, \quad \alpha=\frac{c_{5}}{\sqrt{\log \log T}}, \quad c_{5}>0 .
\end{gathered}
$$

Пусть

$$
I_{1}(t)=\int_{-h_{1}}^{h_{1}} e^{-(u / h)^{2}}|F(t+u)| d u, \quad I_{2}(t)=\left|\int_{-h_{1}}^{h_{1}} e^{-(u / h)^{2}} F(t+u) d u\right| .
$$

Пусть $E$ - множество точек $t$ интервала $(T, T+H)$ таких, что $I_{1}(t)>I_{2}(t)$.

Из определений следует неравенство

$$
I_{3} \leqslant I_{1}+I_{2}
$$

где

$$
I_{1}=\int_{E} I_{1}^{\alpha}(t) d t, \quad I_{2}=\int_{T}^{T+H} I_{2}^{\alpha}(t) d t, \quad I_{3}=\int_{T}^{T+H} I_{1}^{\alpha}(t) d t .
$$

Дословно повторяя соответствующее рассуждение из [17], приходим к неравенству

$$
I_{3} \geqslant c_{6} H h^{\alpha}, \quad c_{6}>0 .
$$

Оценим $I_{1}$ сверху. Применим неравенство Гёльдера:

$$
I_{1}^{2 / \alpha} \ll h^{2}(\mu(E))^{2 / \alpha-1} \int_{T}^{T+H}|F(t)|^{2} d t
$$

Пользуясь следствием 2 и неравенством

$$
\int_{T}^{T+H}|F(t)|^{2} d t \leqslant e \int_{-\infty}^{\infty} \exp \left(-\left(\frac{t-T}{H}\right)^{2}\right)|F(t)|^{2} d t
$$

получаем

$$
\begin{aligned}
\int_{T}^{T+H} & |F(t)|^{2} d t \\
\ll & H \sum_{\lambda_{1} \leqslant P_{0}} \sum_{\lambda_{2} \leqslant P_{0}} \frac{A\left(\lambda_{1}\right)}{\sqrt{\lambda_{1}}} \frac{A\left(\lambda_{2}\right)}{\sqrt{\lambda_{2}}}\left(\frac{\lambda_{2}}{\lambda_{1}}\right)^{-i T} \exp \left(-\frac{1}{4}\left(H \frac{\log \lambda_{2}}{\log \lambda_{1}}\right)^{2}\right) \\
& +H P_{0}^{2} \sum_{P_{0}<\lambda_{1}} \sum_{P_{0}<\lambda_{2}} \frac{A\left(\lambda_{1}\right)}{\lambda_{1}^{3 / 2}} \frac{A\left(\lambda_{2}\right)}{\lambda_{2}^{3 / 2}}\left(\frac{\lambda_{2}}{\lambda_{1}}\right)^{-i T} \exp \left(-\frac{1}{4}\left(H \frac{\log \lambda_{2}}{\log \lambda_{1}}\right)^{2}\right)+O\left(H T^{(3 \varepsilon-1) / 2}\right) .
\end{aligned}
$$

Выделим “диагональные” слагаемые из сумм, стоящих в правой части:

$$
\begin{aligned}
& \int_{T}^{T+H}|F(t)|^{2} d t \\
& \ll H \sum_{\lambda \leqslant P_{0}} \frac{A^{2}(\lambda)}{\lambda}+H\left|\sum_{\lambda_{1}<\lambda_{2} \leqslant P_{0}} \frac{A\left(\lambda_{1}\right)}{\sqrt{\lambda_{1}}} \frac{A\left(\lambda_{2}\right)}{\sqrt{\lambda_{2}}}\left(\frac{\lambda_{2}}{\lambda_{1}}\right)^{-i T} \exp \left(-\frac{1}{4}\left(H \frac{\log \lambda_{2}}{\log \lambda_{1}}\right)^{2}\right)\right| \\
& \quad+H P_{0}^{2}\left|\sum_{P_{0}<\lambda_{1}<\lambda_{2}} \frac{A\left(\lambda_{1}\right)}{\lambda_{1}^{3 / 2}} \frac{A\left(\lambda_{2}\right)}{\lambda_{2}^{3 / 2}}\left(\frac{\lambda_{2}}{\lambda_{1}}\right)^{-i T} \exp \left(-\frac{1}{4}\left(H \frac{\log \lambda_{2}}{\log \lambda_{1}}\right)^{2}\right)\right|+O\left(H T^{(3 \varepsilon-1) / 2}\right) .
\end{aligned}
$$


Оценим первую сумму "недиагональных" слагаемых. Пусть $r$ - рациональное число, большее $1 / X^{2}, \lambda=\lambda_{1}=n \nu_{3} / \nu_{4}, \lambda+r=\lambda_{2}=m \nu_{1} / \nu_{2}$. Зафиксируем $\nu_{1}, \nu_{2}, \nu_{3}, \nu_{4}, r$ и рассмотрим сумму

$$
\begin{array}{r}
S_{1}(U, r)=\sum_{U / 2<n \nu_{1} / \nu_{2} \leqslant U} \frac{A\left(n \nu_{3} / \nu_{4}\right)}{\sqrt{n \nu_{3} / \nu_{4}}} \frac{A\left(n \nu_{3} / \nu_{4}+r\right)}{\sqrt{n \nu_{3} / \nu_{4}+r}}\left(\frac{n \nu_{3} / \nu_{4}+r}{n \nu_{3} / \nu_{4}}\right)^{-i T} \\
\times \exp \left(-\frac{1}{4}\left(H \log \left(1+\frac{r \nu_{4}}{n \nu_{3}}\right)\right)^{2}\right)
\end{array}
$$

где $U \leqslant P_{0}$. Без ограничения общности можно считать, что

$$
H X^{-2} \log ^{-2} T<U, \quad \frac{1}{X^{2}} \leqslant r \leqslant \frac{U}{H} \log ^{2} T .
$$

Пусть $q=\left(\nu_{1} \nu_{4}, \nu_{2} \nu_{3}\right), \nu_{1} \nu_{4}=a q, \nu_{2} \nu_{3}=b q, k=r \nu_{2} \nu_{4} / q$. Считаем, что $k \in \mathbb{N}$, иначе равенство $m \nu_{1} / \nu_{2}-n \nu_{3} / \nu_{4}=r$ не выполняется. Применяя преобразование Абеля и пользуясь неравенством

$$
\begin{aligned}
& \left|\frac{\partial}{\partial n}\left(\frac{1}{\sqrt{n \nu_{3} / \nu_{4}}} \frac{1}{\sqrt{n \nu_{3} / \nu_{4}+r}}\left(\frac{n \nu_{3} / \nu_{4}+r}{n \nu_{3} / \nu_{4}}\right)^{-i T} \exp \left(-\frac{1}{4}\left(H \log \left(1+\frac{r \nu_{4}}{n \nu_{3}}\right)\right)^{2}\right)\right)\right| \\
& \quad \ll\left(\frac{T r}{U^{3}}+\frac{1}{U^{2}}\right) X^{5},
\end{aligned}
$$

получаем

$$
\left|S_{1}(U, r)\right| \ll\left(\frac{T r}{U^{2}}+\frac{1}{U}\right) X^{5}\left|\sum_{\substack{a m-b n=k \\ U / 2<n \leqslant U_{1}}} a(m) a(n)\right|,
$$

где $U_{1} \leqslant U$. Теперь из леммы 6 следует, что

$$
\sum_{U}^{\log T} \sum_{r}^{(U / H) \log ^{2} T}\left|S_{1}(U, r)\right| \ll \frac{T^{15 / 8+8 \varepsilon}}{H^{2}}+\frac{T^{7 / 8+8 \varepsilon}}{H} \ll T^{-\varepsilon},
$$

так как по условию $H \geqslant T^{15 / 16+5 \varepsilon}$.

Таким образом, первая сумма "недиагональных" слагаемых оценена. Оценим вторую. Пусть $P_{0}<U$

$$
\begin{gathered}
S_{2}(U, r)=\sum_{U / 2<n \nu_{1} / \nu_{2} \leqslant U} \frac{A\left(n \nu_{3} / \nu_{4}\right)}{\left(n \nu_{3} / \nu_{4}\right)^{3 / 2}} \frac{A\left(n \nu_{3} / \nu_{4}+r\right)}{\left(n \nu_{3} / \nu_{4}+r\right)^{3 / 2}}\left(\frac{n \nu_{3} / \nu_{4}+r}{n \nu_{3} / \nu_{4}}\right)^{-i T} \\
\times \exp \left(-\frac{1}{4}\left(H \log \left(1+\frac{r \nu_{4}}{n \nu_{3}}\right)\right)^{2}\right) .
\end{gathered}
$$

Рассуждая так же, как при оценке $S_{1}(U, r)$, приходим к неравенству

$$
\sum_{r}^{(U / H) \log ^{2} T}\left|S_{2}(U, r)\right| \ll \frac{U^{15 / 8+7 \varepsilon}}{H^{2} U^{2}}+\frac{U^{7 / 8+7 \varepsilon}}{H U^{2}},
$$

из которого имеем

$$
\sum_{l=1}^{\infty} \sum_{r}^{\left(2^{l-1} P_{0} / H\right) \log ^{2} T}\left|S_{2}\left(2^{l-1} P_{0}, r\right)\right| \ll \frac{T^{15 / 8+8 \varepsilon}}{H^{2} T^{2}} \ll T^{-2-\varepsilon}
$$


Итак,

$$
\int_{T}^{T+H}|F(t)|^{2} d t \ll H \sum_{\lambda \leqslant P_{0}} \frac{A^{2}(\lambda)}{\lambda}+H T^{-\varepsilon} .
$$

Сумма “диагональных" слагаемых оценивается по существу тем же способом, что и в [6], [17].

Оценка имеет вид

$$
\sum_{\lambda \leqslant P_{0}} \frac{A^{2}(\lambda)}{\lambda} \ll(\log T)^{1-2 / h\left(-p_{0}\right)} .
$$

Подробные вычисления содержатся в [12]. Доказано, что

$$
I_{1} \ll(\mu(E))^{1-\alpha / 2} h^{\alpha} H^{\alpha / 2}(\log T)^{\left(1-2 / h\left(-p_{0}\right)\right) \alpha / 2} .
$$

Для $I_{2}$ справедливо неравенство

$$
I_{2}^{2 / \alpha} \ll H^{2 / \alpha-1} \int_{T}^{T+H}\left|\int_{-h_{1}}^{h_{1}} e^{(u / h)^{2}} F(t+u) d u\right|^{2} d t .
$$

И здесь интеграл состоит из “диагональной” и "недиагональной” частей. Сумма "недиагональных" слагаемых оценивается так же, как это сделано при оценке $I_{1}$. Поэтому

$$
I_{2}^{2 / \alpha} \ll H^{2 / \alpha} \sum_{\lambda \leqslant P_{0}} \frac{A^{2}(\lambda) d^{2}(\lambda)}{\lambda}+H^{2 / \alpha-\varepsilon},
$$

где

$$
d(\lambda)=\int_{-h_{1}}^{h_{1}} e^{(u / h)^{2}}\left(\frac{P_{0}}{\lambda}\right)^{i u} d u .
$$

Повторяя рассуждения статьи [17] с учетом (8), получаем

$$
I_{2} \leqslant \frac{1}{2} I_{3}, \quad \mu(E) \geqslant c_{7} H(\log T)^{-\left(1-2 / h\left(-p_{0}\right)\right) \alpha / 2},
$$

где $c_{7}-$ абсолютная постоянная.

Утверждение теоремы прямо следует из оценки $\mu(E)$.

\section{Список литературы}

[1] G. H. Hardy, J. E. Littlewood, "The zeros of Riemann's zeta-function on the critical line", Math. Z., 10:3-4 (1921), 283-317.

[2] A. Selberg, "On the zeros of Riemann's zeta-function", Skr. Norske Vid. Akad. Oslo I, 10 (1942), $59 \mathrm{pp}$.

[3] С. М. Воронин, "О нулях некоторых рядов Дирихле, лежащих на критической прямой”, Изв. АН СССР. Сер. матем., 44:1 (1980), 63-91.

[4] А. А. Карацуба, "О нулях функции Дэвенпорта-Хейльбронна, лежащих на критической прямой", Изв. АН СССР. Сер. матем., 54:2 (1990), 303-315.

[5] С. М. Воронин, А. А. Карацуба, Дзета-функция Римана, Физматлит, М., 1994.

[6] А. А. Карацуба, "О нулях специального вида функций, связанных с рядами Дирихле", Изв. АH СССР. Сер. матем., 55:3 (1991), 483-514.

[7] A. Selberg, "Old and new conjectures about class of Dirichlet series", Collected Papers, Vol. 2, Springer-Verlag, Berlin, 1991, 47-63.

[8] J. B. Conrey, A. Ghosh, "On the Selberg class of Dirichlet series: small degrees", Duke Math. J., 72:3 (1993), 673-693. 
[9] С.А. Гриценко, "О нулях линейных комбинаций специального вида функций, связанных с рядами Дирихле из класса Сельберга”, Изв. РАН. Сер. матем., 60:4 (1996), 3-42.

[10] С. Ленг, Алгебрачческие числа, Мир, М., 1966.

[11] С. А. Гриценко, "О нулях специального вида функций, связанных с $L$-функциями Гекке мнимых квадратичных полей”, Изв. РАН. Сер. матем., 61:1 (1997), 45-68.

[12] И. С. Резвякова, "О нулях линейных комбинаций $L$-функций Гекке на критической прямой”, Изв. РАН. Сер. матем., 74:6 (2010), 183-222.

[13] З. И. Боревич, И. Р. Шафаревич, Теория чисел, Наука, М., 1985.

[14] А.Ф. Лаврик, "Приближенное функциональное уравнение дзета-функции Гекке мнимого квадратичного поля", Матем. заметки, 2:5 (1967), 475-482.

[15] A. Ogg, Modular Forms and Dirichlet Series, New York, W. A. Benjamin, 1969.

[16] С. А. Гриценко, "Об одной аддитивной задаче и ее приложении к проблеме распределения нулей линейных комбинаций $L$-функций Гекке на критической прямой”, Теория чисел, алгебра и анализ, Сборник статей. К 75-летию со дня рождения профессора Анатолия Алексеевича Карацубы, Тр. МИАН, 276, МАИК, М., 2012, 96-108.

[17] А.А. Карацуба, "Новый подход к проблеме нулей некоторых рядов Дирихле", Теория чисел u анализ, Сборник статей. Труды Международной конференции по теории чисел, посвященной 100-летию со дня рождения академика И. М. Виноградова, Тр. МИАН, 207, Наука, М., 1994, 180-196.

[18] С. А. Гриценко, "О распределении норм простых идеалов из заданного класса в арифметических прогрессиях", Труды по теории чисел, Зап. научн. сем. ПОМИ, 322, ПОМИ, СПб., 2005, 45-62. 